\title{
Selective excitation with shaped pulses transported through a fiber using reverse propagation
}

\author{
Monika Pawłowska, Georg Achazi, Nona Rahmat, Alexander Patas, and Albrecht Lindinger \\ Institut für Experimentalphysik, Freie Universität Berlin, Arnimallee 14, D-14195 Berlin, Germany
}

(Received 27 April 2012; published 23 July 2012)

\begin{abstract}
Reverse propagation is a numeric technique that makes it possible to obtain arbitrarily shaped pulses after propagation through a fiber in the nonlinear regime. We apply it to achieve selective two-photon excitation of dyes that have overlapping absorption spectra with pulses transported through the fiber. By comparing both contrast and signal level it is shown that phase and amplitude shaped pulses generated using reverse propagation are superior to pulses with antisymmetric phase despite loss caused by amplitude shaping.
\end{abstract}

DOI: 10.1103/PhysRevA.86.013834

PACS number(s): 42.65.Re, 82.53.Kp, 42.65.Wi, 82.50.Pt

\section{INTRODUCTION}

One of the fields where ultrashort pulses offer an advantage over other optical techniques is nonlinear optical microscopy and imaging. In microscopy, the nonlinear dependence of the signal intensity on the intensity of tightly focused light enables optical sectioning and reduces detrimental out-offocus processes such as photobleaching and photoinduced damage. In addition, infrared lasers have the advantage of falling into the maximum optical transparency window for most biological systems [1].

Several methods used in these fields utilize two-photon processes such as second-harmonic generation [2] and twophoton excited fluorescence [3]. It has been known for some time that the efficiency of such processes can be controlled by shaping the spectral phase of the pulse [4]. More recently, shaped pulses have been incorporated into nonlinear microscopy and imaging. It has been demonstrated that pulse shaping applied to two-photon processes can be used to achieve selective excitation in two-photon microscopy [5-7], suppress unwanted three-photon absorption [8], or provide structural contrast [9].

In parallel, compact fiber-based microscopes are being developed. Devices based on both step-index and photoniccrystal fibers enable measurements using various methods utilizing two- and three-photon processes. Imaging is also possible by using fiber bundles or a scanning mechanism such as a piezoelectric element attached to the fiber end [10].

Combining the techniques mentioned above would allow one to extend the capabilities of fiber-based microscopy. In order to do this one needs the ability to deliver shaped pulses with sufficient pulse energy through a fiber. A problem that has to be overcome to achieve that is the interaction of the femtosecond laser pulses with the fiber. While propagating through an optical fiber, pulses with broad spectrum and high peak power are distorted by dispersion and nonlinear effects, mostly self-phase modulation (SPM).

Several schemes have been proposed to compensate for these effects. If nonlinear effects are negligible, a linear dispersion compensating element such as a grating compressor or a pulse shaper is sufficient. However, this is true only for ultrashort pulses with pulse energy of several picojoules [11]. Another possibility is to use photonic crystal fibers that have ei-

*monika.pawlowska@fu-berlin.de ther a large mode area [12] or an air-filled core [13,14] and thus a smaller nonlinear parameter. This, however, does not eliminate nonlinear effects, but only extends the pulse energy range in which shaped pulses can propagate without distortions.

A more general method of dealing with distortions of the pulse during propagation in the fiber has been proposed by Tsang et al. [15]. It relies on calculating the propagation of the desired pulse shape through the fiber backwards and then generating the required input pulse shape with the help of a pulse shaper. The authors demonstrated the validity of this approach experimentally by characterizing the output pulse, simulating its reverse propagation, and then comparing the simulated pulse to the actual characterized input pulse. They also suggested that a pulse shaper can be used to generate the input pulse shape required to obtain an arbitrary output pulse.

In this paper we apply reverse propagation to selectively optimize the fluorescence of dye molecules with pulses transported through a step-index fiber. We show that amplitudeshaped pulses obtained with reverse propagation allow for selective excitation with contrast and signal better than those achieved with other pulse-shaping methods. The paper is organized as follows: First, the two-photon selective excitation is discussed briefly. Then, the method of obtaining tailored pulses after nonlinear propagation and the experimental setup are described. Finally, the results are presented and discussed.

\section{SELECTIVE FLUORESCENCE EXCITATION}

The efficiency of the selective excitation of two fluorescent species can be described by the contrast factor defined as

$$
\Gamma=\frac{R_{\max }-R_{\min }}{R_{\max }+R_{\min }},
$$

where $R_{\max }$ and $R_{\min }$ are the biggest and the smallest achieved ratios of emission of the two substances. Fluorophores usually have broad absorption spectra which makes it difficult to achieve high contrast unless a narrow excitation source tunable in a wide spectral range is used. However, it is not necessary to achieve $100 \%$ contrast to obtain a good image. If the contrast is sufficient, crosstalk can be eliminated by taking appropriate linear combinations of the two acquired signals as shown in Ref. [6].

One way to achieve selective excitation despite a broad spectrum of the exciting field is coherent control of two-photon excitation. The effective field driving a multiphoton transition is a result of interference between spectral components of the 
pulse field. In particular, for an ultrashort pulse with an electric field spectrum of $E(\omega)=|E(\omega)| e^{i \Phi(\omega)}$ the effective field at a frequency of $2 \omega_{0}$ is [16]

$$
\begin{aligned}
& E^{(2)}\left(2 \omega_{0}\right) \\
& \quad=\int_{-\infty}^{\infty}\left|E\left(\omega_{0}+\Omega\right)\right|\left|E\left(\omega_{0}-\Omega\right)\right| e^{i\left[\Phi\left(\omega_{0}+\Omega\right)+\Phi\left(\omega_{0}-\Omega\right)\right]} d \Omega .
\end{aligned}
$$

If no intermediate level is present, the two-photon excited fluorescence is proportional to $\left|E^{(2)}\left(2 \omega_{0}\right)\right|^{2}$ and can be controlled by shaping the phase of the pulse field $\Phi(\omega)$. In particular, a phase function that is antisymmetric around a certain frequency, $\omega_{0}$, results in a narrow second harmonic field centered at the frequency $2 \omega_{0}$.

When an ultrashort pulse propagates in a fiber, the nonlinear effects modify both its amplitude and phase, so the effective second harmonic field changes as well in a way that in general cannot be predicted without numerical simulation. In a previous paper [17] we have shown that a certain phase, a $\pi$ step, stays antisymmetric despite SPM (although the position of the step changes) which enables selective excitation of narrow transitions. However, this kind of spectral phase is not suitable for distinguishing between broad transitions that are common in biological systems. In this case a different approach is needed.

Another thing that can be done is modulating the spectral amplitude $|E(\omega)|$ to remove unwanted spectral components. The obvious disadvantage is that a significant part of the pulse energy is lost and the price for achieving a good contrast is a low signal level [6]. However, in this paper we show that, if shaped pulses experience nonlinear distortions before reaching the sample, amplitude shaping combined with reverse propagation achieves a higher contrast and at the same time a similar signal level similar to that of phase shaping.

\section{REVERSE PROPAGATION THROUGH THE FIBER}

In the reverse propagation method the spectral phase and amplitude of the input pulse are calculated by simulating the propagation of the pulse through an optical fiber backwards. In order to do that, one has to be able to describe the forward propagation.

For a medium with no second-order electric susceptibility and in the slowly varying envelope approximation the pulse propagation equation has the form [18]

$$
\frac{\partial A}{\partial z}=[\hat{D}+\hat{N}(A)] A,
$$

where $A(z, t)$ is the slowly varying amplitude of the electric field, and $\hat{D}$ and $\hat{N}$ are the linear and the nonlinear operators, respectively. In general, $\hat{D}$ contains loss and chromatic dispersion and $\hat{N}$ terms describing SPM, self-steepening, and the Raman effect. Unless the pulse is linearly polarized and parallel to one of the optical axes of the fiber then birefringence and cross-phase modulation have to be included as well.

To simplify the calculation it is useful to consider which parameters play a role in a given situation and which can be neglected. First of all, for a short single-mode fiber it can be assumed that the dominating source of loss is the coupling into the fiber and the fiber itself is lossless. Second, the dispersion of the fiber does not change rapidly within the relevant spectral range and thus two terms of the Taylor series expansion of the mode-propagation constant $\beta(\omega)$ around the carrier frequency of the pulse, $\beta_{2}$ and $\beta_{3}$, corresponding to the group velocity dispersion (GVD) and third-order dispersion (TOD), are enough to describe it ( $\beta_{1}$ corresponds to a shift of the pulse in time and has no effect on its spectral or temporal shape). Furthermore, our simulations indicate that in the pulse energy regime we consider the higher-order nonlinear effects; that is, self-steeping and intrapulse Raman scattering, are negligible. To sum up, the only parameters we considered in the calculation are $\beta_{2}, \beta_{3}$, and the nonlinear refractive index $n_{2}$. Equation (3) then takes the form

$$
\frac{\partial A}{d z}=\left[\frac{i \beta_{2}}{2} \frac{\partial^{2}}{d t^{2}}-\frac{\beta_{3}}{6} \frac{\partial^{3}}{d t^{3}}+i \frac{\omega_{0} n_{2}}{c A_{\mathrm{eff}}}|A|^{2}\right] A,
$$

where $A_{\text {eff }}$ is the effective mode area (in many cases the nonlinear parameter $\gamma=n_{2} \omega_{0} / c A_{\text {eff }}$ is used instead). In the following we assume that $n_{2}$ and $A_{\text {eff }}$ are constant within the pulse bandwidth.

Equation (3) can be used to describe the propagation of a pulse in both directions. Backwards propagation means reversing the sign of $z$, which is in turn mathematically equivalent to reversing the signs of the parameters $\beta_{2}, \beta_{3}$, and $n_{2}$ in Eq. (4). Usually this means that the same code which is used for numerical forward propagation can be also used for reverse propagation simply by changing the signs of the input parameters. We used the split-step routine taken from the LAB2 package [19] for LabVIEW mainly because it was convenient to integrate it with the existing code for converting given pulse shapes into transmission and phase patterns that can be applied on the pulse shaper.

The most important additional thing to consider while implementing the reverse propagation algorithm to apply it to pulse shaping is accounting for the spectral amplitude shape. In general, by reverse propagation of a given output pulse one obtains an input pulse that has a spectral amplitude different than that of the available laser pulse. The required spectrum can be obtained by amplitude shaping, but then the pulse energy changes and the performed simulation is not valid anymore. We solved this problem by assuming a fixed loss due to amplitude shaping (or in other words a fixed overall shaper transmission) and using a modified pulse energy value for the simulation. The obtained transmission profile is then scaled down to fit the assumed total transmission. If there is a need for scaling up — which is not possible with a shaper-the program indicates an error. On a standard PC the whole procedure takes a few seconds per pulse shape. In order to shorten the time of the actual measurement, we calculate the set of pulses beforehand and then load the pattern that has to be written on the modulator from a file. This way the only limitation is the modulator switching time.

Contrary to adaptive optimization, which can produce desired pulses with no or very little knowledge of the fiber properties, reverse propagation requires precise knowledge of the parameters that appear in Eq. (4). Simply assuming the parameters of bulk fused silica is not sufficient. First of all, the waveguide dispersion is not negligible [20]. Second, the known values for $n_{2}$ for femtosecond pulses at $800 \mathrm{~nm}$ are 
scarce and not reliable enough as it has been shown that the nonlinearity of different samples of fused silica can differ by as much as $30 \%$ [21].

To our knowledge there are no exact data for the fiber used in this experiment so we had to measure the dispersion and nonlinear refractive index ourselves. The dispersion was measured using a procedure first described in Ref. [20]. It relies on shaping the spectral phase of a pulse entering the fiber so that the pulse length after propagation through the fiber is minimized and the dispersion is calculated from the optimal phase. The advantage of this method is that dispersion properties are measured exactly in the spectral range that the laser pulse is covering. Our method for determining $n_{2}$ relies on simulating spectral narrowing for negatively chirped pulses. More precisely, we measure the spectrum after the fiber and then compare the spectrum obtained by reverse propagation with different values of $n_{2}$ with the actual input spectrum.

\section{EXPERIMENTAL SETUP}

The experimental setup is shown in Fig. 1. The laser source is a Ti:sapphire femtosecond oscillator (Mira, Coherent) delivering pulses at the repetition rate of $76 \mathrm{MHz}$ with a $28-\mathrm{nm}$ spectral width centered at $805 \mathrm{~nm}$. The pulse shaper consists of two diffraction gratings with 1200 lines $/ \mathrm{mm}$, two cylindrical lenses with $250-\mathrm{mm}$ focal length in a standard $4 f$ zero dispersion compressor configuration and a double liquid crystal array spatial light modulator (SLM-640, CRi) placed in the Fourier plane. The resolution of this setup is $0.27 \mathrm{~nm} /$ pixel. Together with a polarizer placed after the second grating the setup enables phase shaping and amplitude shaping. A phase offset is determined to remove the chirp of the laser pulse and compensate for the dispersion of the optical elements in the setup so that the pulse is transform limited when no additional pattern is written on the modulator.

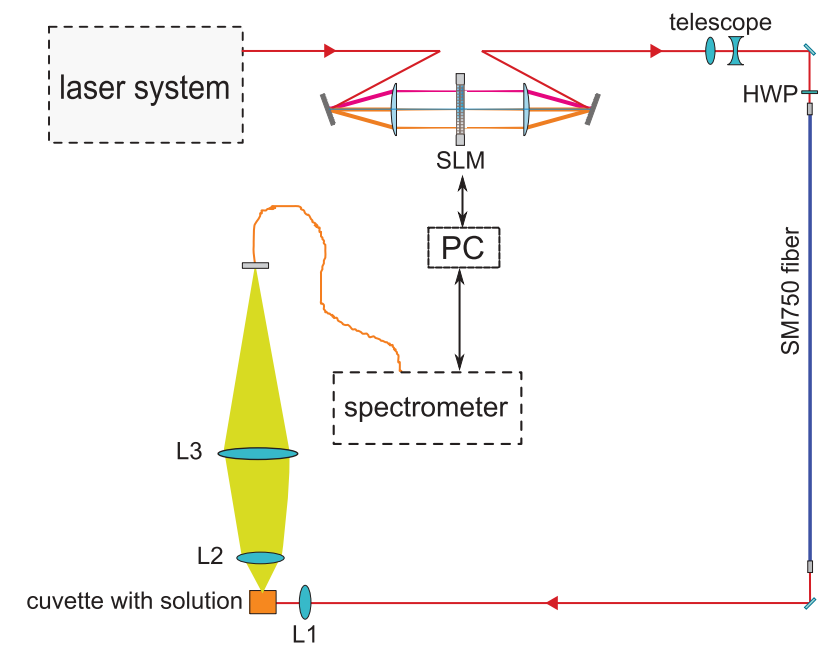

FIG. 1. (Color online) Experimental setup. A laser pulse is shaped by a spatial light modulator (SLM) placed in a $4 f$ setup. A half-wave plate (HWP) rotates the polarization to coincide with an optical axis of the fiber. After propagating through the fiber the beam is focused in a cuvette with lens L1 and the fluorescence is collected by lenses L2 and L3 into a spectrometer.
The laser beam is then coupled into a 1-m-long silica single-mode step index optical fiber with a 5.3- $\mu \mathrm{m}$ mode field diameter (SM750, Fibercore Limited) using a standard collimator (F-C5-F3-780, Newport) directly attached to the fiber FC/PC connector. The pulse energy after the fiber is limited to about $0.8 \mathrm{~nJ}$ because of the transmission of the shaper setup (mainly grating reflectivity) and the efficiency of coupling into the fiber. The latter was around $35 \%$ even when a telescope was used to optimize the beam waist at the fiber input.

For the measurement of the fiber dispersion the pulse energy was reduced using a neutral density filter to suppress nonlinear effects. After verifying that adding further phase terms in the optimization does not further reduce the pulse duration, $\beta_{2}(\omega)$ is expressed using the first two Taylor terms. The obtained GVD value $\left(41.0 \mathrm{fs}^{2} / \mathrm{mm}\right)$ is about $10 \%$ greater than the value for bulk fused silica $\left(35.8 \mathrm{fs}^{2} / \mathrm{mm}\right)$. This indicates that the waveguide contribution has the same sign as the material contribution, which is consistent with calculations for single-mode fibers [22,23] as well as with the measurement for a similar fiber included in Ref. [20]. Also the TOD value that we obtained $\left(153 \mathrm{fs}^{3} / \mathrm{mm}\right)$ is different from the one for bulk material $\left(27.8 \mathrm{fs}^{3} / \mathrm{mm}\right)$.

For the nonlinear refractive index we obtained $n_{2}=2.6 \times$ $10^{-20} \mathrm{~m}^{2} \mathrm{~W}^{-1}$, which is consistent with previous measurements [21]. The uncertainty of this measurement results from uncertainties of the other parameters used in the simulation of spectral narrowing: the fiber dispersion, the spectral phase and amplitude of the pulse, and the pulse intensity measurement. However, if the same power meter is used all along and only SPM is considered, as in this case, a systematic error of intensity measurement has no influence on the pulse shaping because the parameter determining the strength on nonlinear effects is in fact the product of $n_{2}$ and pulse intensity.

The fiber parameters considered above are sufficient to calculate the input pulse shape that yields the desired output pulse shape. The next step is determining the shaper modulation function - transmission and phase filter-required to generate the calculated pulse shape. For this the spectral amplitude and phase of the available laser pulse must be known. The overall intensity is measured with a power meter. When measuring pulse spectrum one has to keep in mind possible losses during coupling into the fiber. Apart from a constant loss that reduces the pulse energy we observe spectrally dependent loss caused by space-time coupling effects in the shaper. Space-time coupling is an inherent effect caused by coupling between the temporal phases of the pulse components and their wave vectors [24]. To investigate its influence on the spectrum coupled into the fiber we placed a $10-\mathrm{cm}$-long piece of SF10 glass to stretch the pulse temporally and thus eliminate nonlinear effects that also modulate the spectrum. We found that the biggest contribution in our case is the quadratic phase of around $-5 \times 10^{4} \mathrm{fs}^{2}$ that is needed to compensate the GVD of the fiber. It causes linear spatial chirp and thus limits the spectral bandwidth coupled into the fiber to about $20 \mathrm{~nm}$. Finally, autocorrelation is measured to ensure that the pulse is transform limited and therefore the phase is flat.

The selective excitation is demonstrated in a mixture of two dyes, Coumarin 1 (also known as Coumarin 460 or Coumarin 47) and Rhodamine B, dissolved in ethanol. The dyes were 

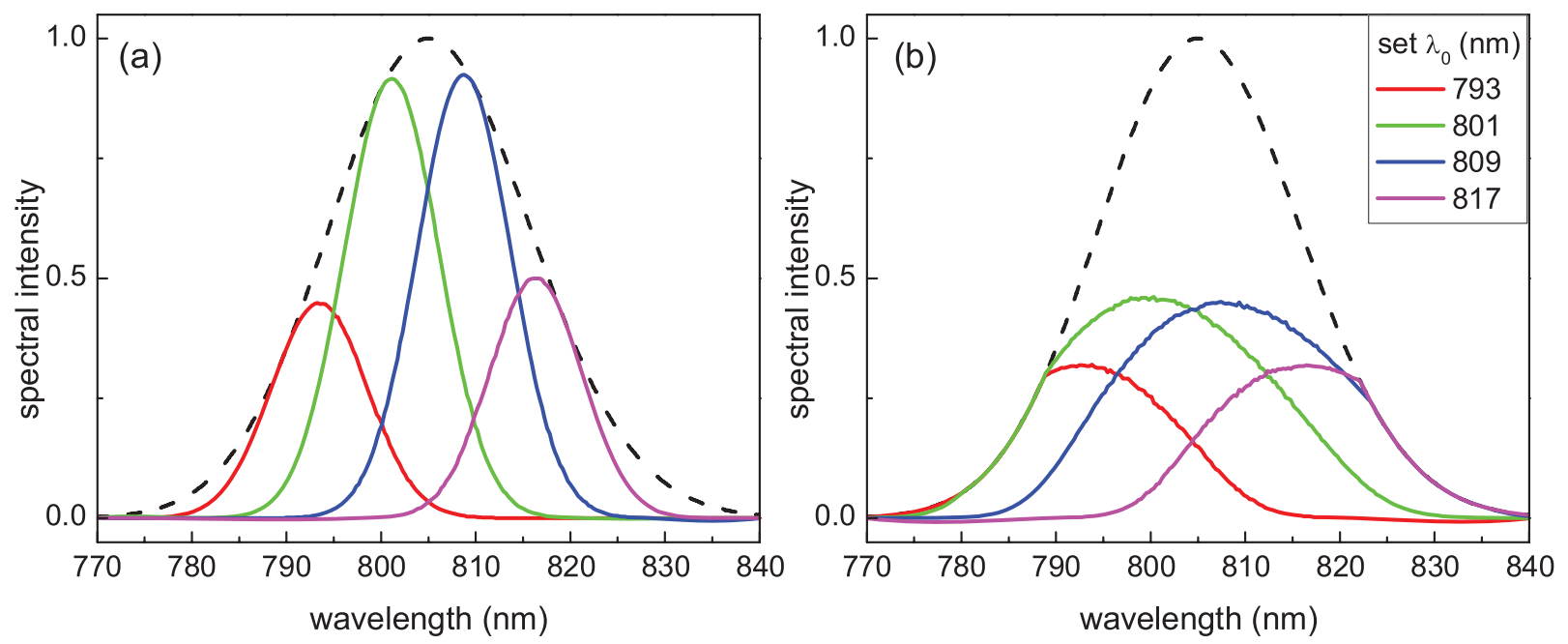

FIG. 2. (Color online) Generating short pulses with changing central wavelength by reverse propagation. (a) Desired spectral amplitude after the fiber. (b) Calculated input spectral amplitude for pulses with 12-nm spectral width and central wavelengths: 793, 801, 809, and 817 nm. Dashed line: Pulse spectrum before the shaper.

chosen because they satisfy two conditions. First of all, they both absorb at $400 \mathrm{~nm}$, which is the second harmonic of our pulse, but for Coumarin 1 the maximum of absorption is blueshifted from this wavelength (see Ref. [25]) and for Rhodamine $B$ the maximum of absorption is red-shifted (to $420 \mathrm{~nm}$ according to Ref. [26]). Second, because we tested the method in solution we had to use two dyes with different fluorescence spectra, which is the case for Coumarin 1 and Rhodamine B [see inset in Fig. 4(a)], but in an actual imaging setup where the fluorophores are present in different concentrations in different regions of the sample this is obviously not a requirement.

The concentration of dyes in the solution was $13 \mathrm{mM}$ for Coumarin 1 and $0.33 \mathrm{mM}$ for Rhodamine B. The proportions were chosen so that the ratio of emission for an unshaped pulse was close to 1 . The fluorescence was collected with a lens and coupled into a spectrometer and then the two spectral regions corresponding to the fluorescence of each dye were integrated. In order to minimize the reabsorption of Coumarin 1 fluorescence by Rhodamine B the cuvette with solution was placed in such a away that the excited volume was as close to the glass surface as possible.

\section{RESULTS}

In this section we demonstrate selective fluorescence excitation with pulses obtained by reverse propagation. Our approach is to use amplitude shaping in addition to phase shaping to generate a spectrally narrow pulse with shifting central wavelength (in the range limited by the input pulse spectral width). Because of spectral narrowing the pulses before the fiber have to be broader than the desired output pulses and the spectral phase has to precompensate for chromatic dispersion
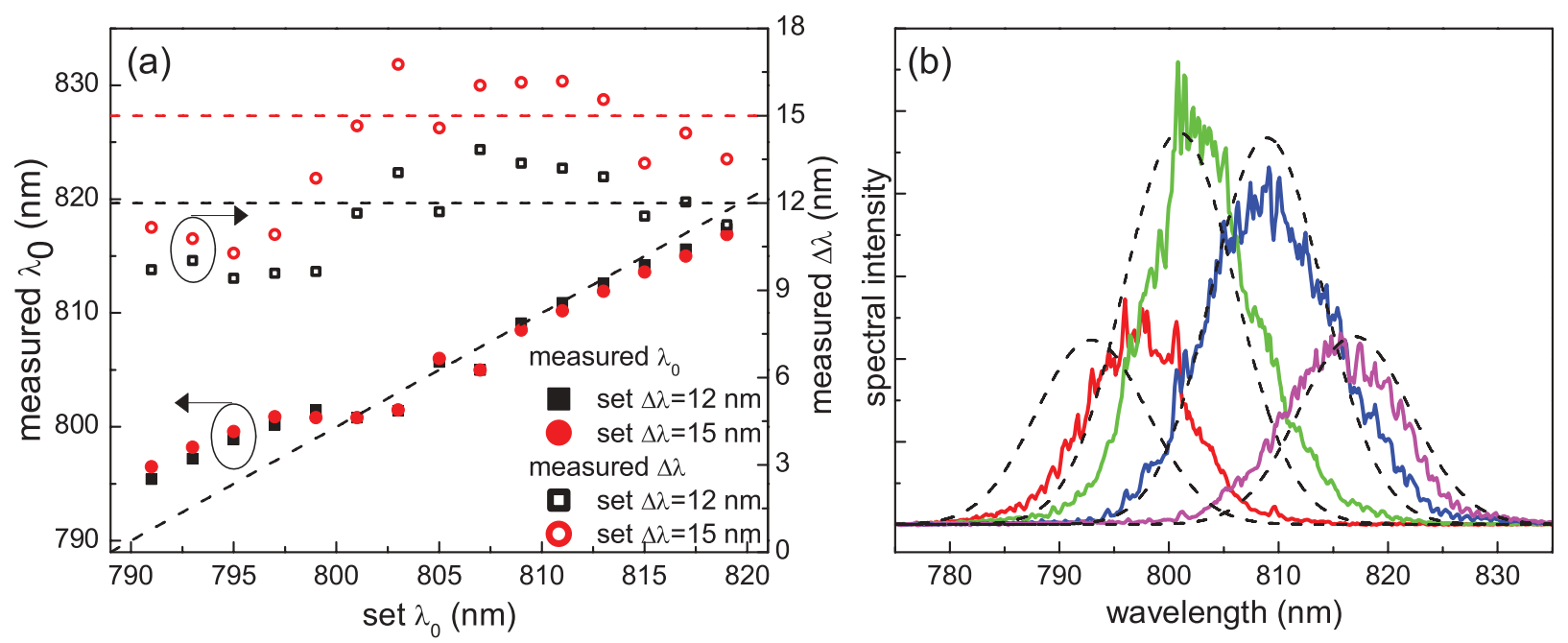

FIG. 3. (Color online) Short pulses with changing central wavelength obtained by reverse propagation. (a) Measured central wavelength (solid symbols) and spectral width (open symbols) for pulses with spectral width set to $12 \mathrm{~nm}$ (black squares) and $15 \mathrm{~nm}$ (red circles). Set central wavelength and spectral width marked with dashed lines. (b) Solid lines: Measured spectra after propagation through the fiber for 12-nm spectral width and central wavelengths: 793, 801, 809, and $817 \mathrm{~nm}$. Dashed line: Expected spectrum. 

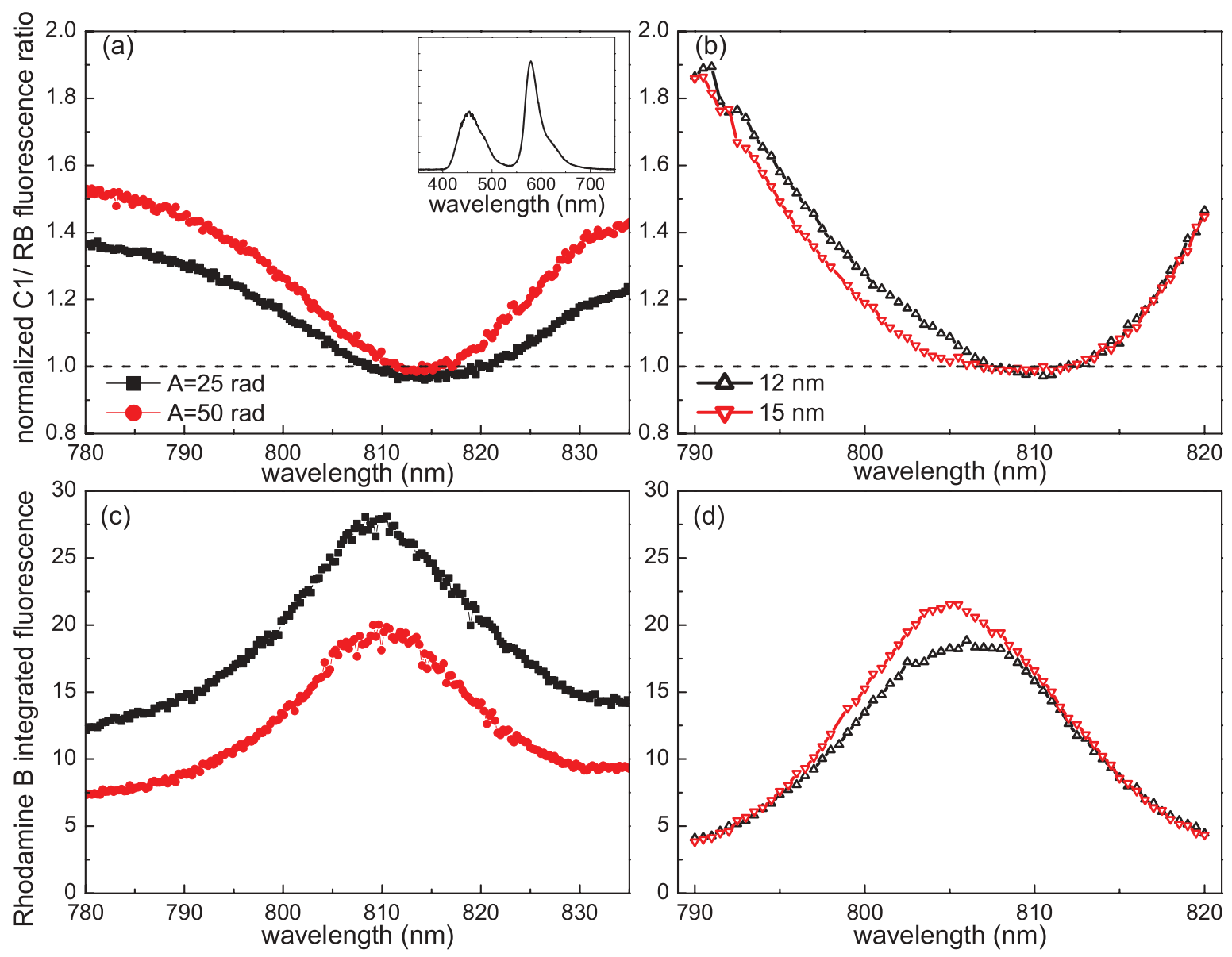

FIG. 4. (Color online) (a) Coumarin 1 (C1) to Rhodamine B (RB) normalized fluorescence ratio for phase-shaped pulses with sinusoidal phase with $b=0.055 \mathrm{~nm}^{-1}$ and changing $\lambda_{0}$. Inset: Joint fluorescence spectrum for an unshaped pulse. (b) $\mathrm{C} 1$ to $\mathrm{RB}$ fluorescence ratio for pulses with changing central wavelengths obtained by reverse propagation. (c) and (d) Integrated RB fluorescence for pulses from panels (a) and (b), respectively.

and nonlinear phase shift. Examples of calculated input pulse spectra are shown in Fig. 2(b). Next, we verify that the desired pulse shapes are generated correctly. The systematic variation of the central wavelength for two different spectral widths, 12 and $15 \mathrm{~nm}$, is presented in Fig. 3(a). Figure 3(b) shows some example spectra measured after the fiber.

In previous work concerning selective excitation of fluorophores with shaped pulses phase-only modulated pulses were used $[5,6]$. Here we compare this approach to selective excitation with amplitude-shaped pulses obtained by reverse propagation for the case when pulses propagate through a fiber in the nonlinear regime. For that we use phase-shaped pulses with the sinusoidal phase function $\Phi(\lambda)=A \sin \left[b\left(\lambda-\lambda_{0}\right)\right]$ combined with dispersion compensation.

The results are shown in Fig. 4. First, the ratio of Coumarin 1 to Rhodamine B fluorescence normalized with the value for an unshaped short pulse (with phase optimized to achieve minimal pulse duration after the fiber) is shown. Selective excitation using pulses with a sinusoidal spectral phase with changing $\lambda_{0}, b=0.055 \mathrm{~nm}^{-1}$, and two different values of $A$ [Fig. 4(a)] is compared to excitation with short Gaussian pulses with a changing central wavelength [Fig. 4(b)]. First of all, it can be seen that the contrast [as defined by Eq. (1)] achieved by shifting the central wavelength is much higher, $\Gamma=0.33$ compared to $\Gamma=0.22$, for the sinusoidal phase with amplitude $A=50$. However, in both cases it was possible to increase the ratio significantly, but not to decrease it. This means that no pulse shape that significantly enhances Rhodamine B fluorescence compared to Coumarin 1 was found, possibly because the absorption of Rhodamine $\mathrm{B}$ is fairly constant in the spectral range covered by the pulse bandwidth.

Figures 4(c) and 4(d) show the corresponding Rhodamine B fluorescence signal. Remarkably, although the energy of the pulses with shifting central wavelength was reduced due to amplitude shaping by a factor between 2 and 7 (depending on the spectral width and detuning from the central wavelength of the laser pulse), the fluorescence signal is comparable to the signal obtained with phase-shaped pulses generated without reverse propagation. This can be explained by the pulses with shifting central wavelengths being shorter, which results in greater efficiency of two-photon excitation. A similar effect can be seen if sinusoidal phases with different values of $A$ are compared. A larger value of $A$ corresponds to a stronger phase modulation, which results in less signal, but more contrast. The values used in the presented measurements were chosen as a compromise. As a side effect, if a lower pulse energy is required for the same 

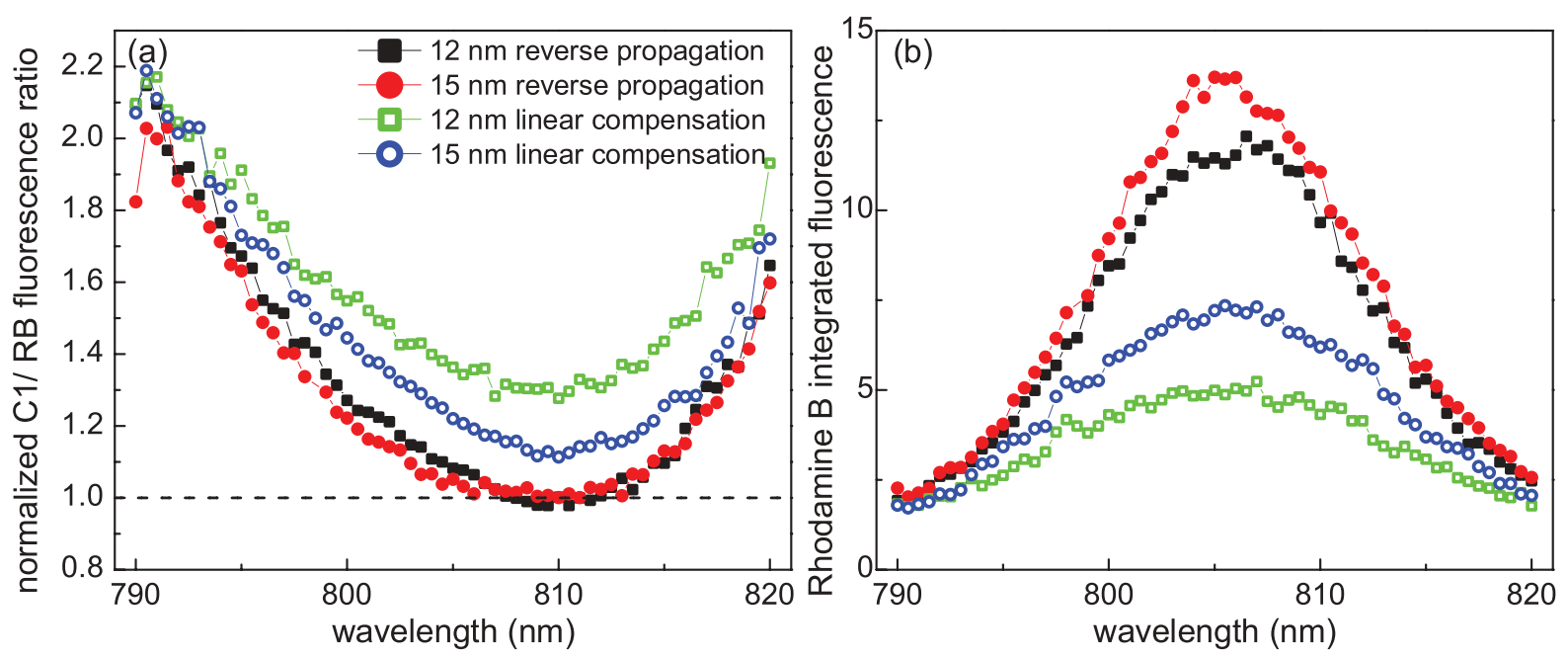

FIG. 5. (Color online) (a) Coumarin 1 (C1) to Rhodamine B (RB) normalized fluorescence ratio for pulses with changing central wavelengths and spectral width set to $12 \mathrm{~nm}$ (squares) or $15 \mathrm{~nm}$ (circles): reverse propagation (solid symbols) compared to linear compensation (open symbols). (b) Corresponding integrated RB fluorescence.

amount of signal, as is the case with the amplitude-shaped pulses, sample damage by heating can be reduced.

In a separate measurement we compared pulses with shifting central wavelengths generated by two methods: reverse propagation and solely linear compensation to confirm that the improvement is due to reverse propagation and not amplitude shaping alone. In the case of linear compensation the amplitude modulation by nonlinear effects is not accounted for, so the spectral amplitude of the input pulses is the same as that of the desired output pulses (see examples in Fig. 2). The results are shown in Fig. 5. For pulses obtained by reverse propagation the contrast that can be calculated from the fluorescence ratio shown in Fig. 5(a) is slightly larger. More importantly, the fluorescence signal shown in Fig. 5(b) is significantly better (by more than a factor of 2). To make the comparison meaningful, the shaper transmission was adjusted so that the pulse energy for a pulse with a given central wavelength was the same in both cases, which was verified using a photodiode. However, reverse propagation takes into account that the amount of phase modulation differs depending on the pulse spectral amplitude. Therefore the pulses are shorter than those obtained by linear compensation.

\section{DISCUSSION AND SUMMARY}

The measurements presented above show that reverse propagation is superior to other commonly used methods, as it achieves simultaneously more contrast and signal. In principle, comparable results could be also obtained by other methods. Every pulse shape that is possible to obtain by reverse propagation could be obtained by characterizing the pulse after the fiber and adapting the input pulse shape iteratively until the desired output pulse is reached. Another possibility is a multiobjective optimization algorithm with contrast and signal as optimization goals. However, these methods are not practical for two-photon microscopy. In the first case a bulky pulse characterization setup is required. For adaptive contrast optimization one needs to know a priori where the different areas of the sample are in order to have an observable that can be optimized. Compared to that, reverse propagation is quite simple. The fiber can be characterized before attaching it to the microscope and very little knowledge of the investigated sample itself is required.

The pulse spectral bandwidth in our measurements, especially considering the spectral narrowing, was quite modest. One can expect better results with broader pulses. To see what contrast could be achieved with the two dyes we used we repeated the measurement without the fiber, with the full available bandwidth of $28 \mathrm{~nm}$. The best achieved value with amplitude shaping was $\Gamma=0.44$. In our case the bandwidth after the fiber was limited by the bandwidth of the laser itself. When using a broadband laser one has to choose the fiber carefully, but it has been reported that a pulse spectrum as broad as $120 \mathrm{~nm}$ can fully be transmitted (although with some modulations of unclear origin) through a polarizationmaintaining single-mode fiber [27].

In summary, reverse propagation is a promising method for applications where selective two-photon excitation takes place in setups containing optical fibers, such as in vivo two-photon microscopy and imaging.

\section{ACKNOWLEDGMENTS}

We thank Professor Ludger Wöste for support. M.P. acknowledges the financial support of the Studienstiftung des deutschen Volkes.
[1] K. Isobe, A. Suda, M. Tanaka, H. Hashimoto, F. Kannari, H. Kawano, H. Mizuno, A. Miyawaki, and K. Midorikawa, IEEE J. Sel. Top. Quantum Electron. 16, 767 (2010).
[2] R. Hellwarth and P. Christensen, Opt. Commun. 12, 318 (1974).

[3] W. Denk, J. H. Strickler, and W. W. Webb, Science 248, 73 (1990). 
[4] D. Meshulach and Y. Silberberg, Nature (London) 396, 239 (1998).

[5] I. Pastirk, J. D. Cruz, K. Walowicz, V. Lozovoy, and M. Dantus, Opt. Express 11, 1695 (2003).

[6] J. P. Ogilvie, D. Débarre, X. Solinas, J. Martin, E. Beaurepaire, and M. Joffre, Opt. Express 14, 759 (2006).

[7] G. Labroille, R. S. Pillai, X. Solinas, C. Boudoux, N. Olivier, E. Beaurepaire, and M. Joffre, Opt. Lett. 35, 3444 (2010).

[8] V. V. Lozovoy, I. Pastirk, K. A. Walowicz, and M. Dantus, J. Chem. Phys. 118, 3187 (2003).

[9] P. Schön, M. Behrndt, D. Ait-Belkacem, H. Rigneault, and S. Brasselet, Phys. Rev. A 81, 013809 (2010).

[10] L. Fu and M. Gu, J. Microsc. 226, 195 (2007).

[11] F. Weise, M. Pawłowska, G. Achazi, and A. Lindinger, J. Opt. 13, 075301 (2011).

[12] T. Le, G. Tempea, Z. Cheng, M. Hofer, and A. Stingl, Opt. Express 17, 1240 (2009).

[13] W. Göbel, A. Nimmerjahn, and F. Helmchen, Opt. Lett. 29, 1285 (2004).

[14] F. Weise, G. Achazi, and A. Lindinger, Phys. Rev. A 82, 053827 (2010).

[15] M. Tsang, D. Psaltis, and F. G. Omenetto, Opt. Lett. 28, 1873 (2003).
[16] B. Broers, L. D. Noordam, and H. B. van Linden van den Heuvell, Phys. Rev. A 46, 2749 (1992).

[17] M. Pawłowska, A. Patas, G. Achazi, N. Rahmat, F. Weise, and A. Lindinger, J. Opt. Soc. Am. B 29, 833 (2012).

[18] G. Agrawal, Nonlinear Fiber Optics (Academic Press, San Diego, 2007).

[19] B. Schmidt, M. Hacker, G. Stobrawa, and T. Feurer, LAB2-a virtual femtosecond laser lab.

[20] G. Stobrawa, M. Hacker, R. Netz, M. Bischoff, and R. Sauerbrey, Appl. Phys. B 76, 333 (2003).

[21] S. Santran, L. Canioni, L. Sarger, T. Cardinal, and E. Fargin, J. Opt. Soc. Am. B 21, 2180 (2004).

[22] J. K. Ranka, R. S. Windeler, and A. J. Stentz, Opt. Lett. 25, 25 (2000).

[23] D. Mogilevtsev, T. A. Birks, and P. S. J. Russell, Opt. Lett. 23, 1662 (1998).

[24] F. Frei, A. Galler, and T. Feurer, J. Chem. Phys. 130, 034302 (2009).

[25] J. P. Ogilvie, K. J. Kubarych, A. Alexandrou, and M. Joffre, Opt. Lett. 30, 911 (2005).

[26] N. S. Makarov, M. Drobizhev, and A. Rebane, Opt. Express 16, 4029 (2008).

[27] A. M. Larson and A. T. Yeh, Opt. Express 16, 14723 (2008). 